\title{
Completing rule bases in symbolic domains by analogy making
}

\author{
Henri Prade ${ }^{1}$ Steven Schockaert ${ }^{2}$ \\ ${ }^{1}$ Institut de Recherche en Informatique de Toulouse (IRIT), Université Paul Sabatier, Toulouse, France \\ ${ }^{2}$ Ghent University, Department of Applied Mathematics and Computer Science, Ghent, Belgium
}

\begin{abstract}
The paper considers the problem of completing a set of parallel if-then rules that provides a partial description of how a conclusion variable depends on the values of condition variables, where each variable takes its value among a finite ordered set of labels. The proposed approach does not require the use of fuzzy sets for the interpretation of these labels or for defining similarity measures, but rather relies on the extrapolation of missing rules on the basis of analogical proportions that hold for each variable between the labels of several parallel rules. The analogical proportions are evaluated for binary and multiple-valued variables on the basis of a logical expression involving Łukasiewicz implication. The underlying assumption is that the mapping partially specified by the given rules is as regular as suggested by these rules. A comparative discussion with other approaches is presented.
\end{abstract}

Keywords: Commonsense reasoning; analogical proportions; incomplete information; if-then rules

\section{Introduction}

Since information is often partially missing in practice, or is at least not explicitly stated, reasoning under incomplete information is a problem that has been much studied in different artificial intelligence settings during the last three decades. Still, diverse instances of this problem class exist. The setting of default rules with exceptions which are to be applied to an incompletely described situation has especially been studied in non-monotonic reasoning (see e.g., $[1,2]$ ). However, there are other forms of reasoning under incomplete information, such as reasoning with sparse parallel (fuzzy) rules (see e.g., $[3,4,5])$.

In the following, we consider the problem of reasoning from a collection of parallel if-then rules of the form "if $X_{1}$ is $A_{1}$ and $\ldots$ and $X_{n}$ is $A_{n}$ then $Y$ is $B$ " where the $A_{i}$ 's and the $B$ 's are labels belonging to ordered domains; for instance, the labels associated with the domain of $X_{i}$ could be 'small', 'medium', and 'large'. This rule base may be incomplete in the sense that for some combination of the labels of the condition variables $X_{i}$, there may not be a corresponding rule. Such a problem may be handled by considering that the $A_{i}$ 's and the $B$ 's are represented by fuzzy sets. Still, in a computing with words [6] perspective, one may not require the use of fuzzy sets for the interpretation of linguistic terms (as in the early proposal made in [7]), or at least one may want that the inference be symbolically processed independently from any semantic interpretation associated with particular fuzzy sets [8].

This means that we look for a qualitative approach which does not rely on measuring similarity, but rather assumes that the mapping from the $X_{i}$ 's to $Y$ which is partially described by the set of available rules, is sufficiently "regular" for extrapolating missing rules. Such problems are often encountered when stating recommendations, or multiple criteria evaluations. For instance, specifying the daily requirements of the human body in calories may involve such rules as "if the amount of physical activity is small and the age is young, the calorie needs are medium". As another example, we may have rules such as "if the price is moderately expensive and the surface is large and the location is rather good, the apartment to rent is satisfactory". Clearly, when there are several conditions and the number of possible labels for each attribute increases, the number of rules rapidly becomes prohibitive, and a mechanism for completing an incomplete rule base becomes of interest.

In the following we propose an approach based on a recently developed formal model of analogical proportions $[9,10]$ in order to cope with this problem. The paper is organized as follows. The next section provides a short background on the evaluation of analogical proportions for binary and multiplevalued features. We then explain how this tool may be useful for extrapolating rules, discuss several aspects of the approach, before concluding.

\section{Analogical proportions}

An analogical proportion is a statement of the form " $a$ is to $b$ as $c$ is to $d$ ", and is usually denoted by $(a: b:: c: d)$. A formal study of analogical proportions has first been proposed by Lepage in [11] in a computational linguistics perspective some years ago, and has further been developed in [12]. These authors proposed a definition of analogical proportions, which was restated in a different, simpler but equivalent way in [9]. The underlying idea is that 


\begin{tabular}{c|cccc} 
& $a_{i}$ & $b_{i}$ & $c_{i}$ & $d_{i}$ \\
\hline 1 & 1 & 1 & 1 & 1 \\
2 & 0 & 0 & 0 & 0 \\
3 & 1 & 1 & 0 & 0 \\
4 & 0 & 0 & 1 & 1 \\
5 & 1 & 0 & 1 & 0 \\
6 & 0 & 1 & 0 & 1
\end{tabular}

Table 1: The 6 cases where an analogical proportion holds

the analogical proportion $(a: b:: c: d)$ holds if and only if " $a$ differs from $b$ as $c$ differs from $d$ " and " $b$ differs from $a$ as $d$ differs from $c$ " (see [10]).

\subsection{Binary case}

Let us assume that $a, b, c$ and $d$ denote vectors of the values of $n$ features $\mathcal{F}_{1}, \ldots, \mathcal{F}_{n}$, which are assumed to be binary valued for the moment, i.e., $a=\left(a_{1}, \ldots, a_{n}\right), b=\left(b_{1}, \ldots, b_{n}\right), c=\left(c_{1}, \ldots, c_{n}\right)$, and $d=\left(d_{1}, \ldots, d_{n}\right)$, with $a_{i}, b_{j}, c_{k}, d_{l} \in\{0,1\}$. Let $A=\left\{\mathcal{F}_{i} \mid a_{i}=1\right\}, B=\left\{\mathcal{F}_{j} \mid b_{j}=1\right\}, C=\left\{\mathcal{F}_{k} \mid c_{k}=\right.$ $1\}$, and $D=\left\{\mathcal{F}_{l} \mid d_{l}=1\right\}$. Then the analogical proportion can be equivalently stated

- in a set-valued form. Let $\bar{A}$ denote the complementary set of $A$ in $\left\{\mathcal{F}_{1}, \ldots, \mathcal{F}_{n}\right\}$ and $A-B=A \cap \bar{B}$. Then

Definition $1(a: b:: c: d)$ holds if

$$
(A-B=C-D) \text { and }(B-A=D-C) .
$$

Thus, " $A: B$ " refers to the set operation that transforms $A$ into $B$ by deleting the elements of $A-B$ and adding the elements of $B-A$.

- in a Boolean logic form. Let $\neg$ and $\wedge$ denote negation and conjunction, $\rightarrow$ and $\equiv$ the associated implication and equivalence $(x \rightarrow y=\neg(x \wedge \neg y)$, $x \equiv y=(x \rightarrow y) \wedge(y \rightarrow x))$. Then it can be shown that the logical counterpart of the above definition writes:

Definition $2(a: b:: c: d)$ holds if $\forall i=$ $1, \ldots, n$,

$$
\left(\left(a_{i} \rightarrow b_{i}\right) \equiv\left(c_{i} \rightarrow d_{i}\right)\right) \wedge\left(\left(b_{i} \rightarrow a_{i}\right) \equiv\left(d_{i} \rightarrow c_{i}\right)\right) .
$$

These two equivalent definitions clearly satisfy the three postulates generally considered as being characteristic of analogical proportions:

- $(a: b:: a: b)$ and $(a: a:: b: b)$ (identity)

- $(a: b:: c: d) \Rightarrow(c: d:: a: b)$ (symmetry)

- $(a: b:: c: d) \Rightarrow(a: c:: b: d)$ (central permutation).

The six patterns that make the quaternary connective $\left(a_{i}: b_{i}:: c_{i}: d_{i}\right)$ true are given in Table 1 . For the 10 other 4 -tuples of binary values, $(a: b:: c: d)$ is false. As can be seen, the analogical proportion $\left(a_{i}: b_{i}:: c_{i}: d_{i}\right)$ holds either when $a_{i}$ and $b_{i}$ (resp. $c_{i}$ and $d_{i}$ ) are identical, or when the changes from $a_{i}$ to $b_{i}$, and from $c_{i}$ to $d_{i}$ take place in the same direction simultaneously (see the two last lines of Table 1).

It is worth noticing in Table 1 that changing the 1's into 0's leaves the table unchanged, which expresses that the evaluation of an analogical proportion does not depend on the way it is encoded. Namely, it would be the same, when describing a situation, to state that a property holds or that its negation is false.

\subsection{Multiple-valued case}

The evaluation of analogical proportions has been extended to multiple valued situations, i.e., when $a_{i}, b_{j}, c_{k}, d_{l} \in[0,1]$. It has been advocated in [13] that an appropriate extension of Definition 2 is obtained by choosing

$$
\begin{aligned}
& \text { - } x \wedge y=\min (x, y) \\
& \text { - } x \rightarrow y=\min (1,1-x+y) \\
& \text { - } x \equiv y=1-|x-y|
\end{aligned}
$$

Then it can be checked that $\left(a_{i}: b_{i}:: c_{i}: d_{i}\right)=1$ if and only if $\left|a_{i}-b_{i}\right|=\left|c_{i}-d_{i}\right|$ and $a_{i} \leq b_{i} \Leftrightarrow$ $c_{i} \leq d_{i}$. This expresses that the amount of change from $a_{i}$ to $b_{i}$, and from $c_{i}$ to $d_{i}$ is the same and is in the same direction. In the particular case where only the 3 values $0, \frac{1}{2}, 1$ are used, the analogical proportion holds at the degree 1 only for the 19 patterns given in Table 2 .

It is important to notice that the above operations make sense on finite ordered scales [14, 15, 16], which makes them more compatible with a qualitative reading. Still, the use of Eukasiewicz implication (and the associated equivalence) requires that the distance between two successive levels in the scale be always the same. For instance, the patterns in Table 2 do not require a numerical interpretation. One may as well use the ordered scale $\{\top, \alpha, \perp\}$ instead of $\left\{1, \frac{1}{2}, 0\right\}$. However, considering that the analogical proportion ( $\top: \alpha:: \alpha: \perp$ ) holds implicitly assumes that $\top$ differs from $\alpha$ as $\alpha$ from $\perp$, in a strict reading. One might also use a more permissive view of graded analogical proportions that would be defined from acceptable patterns directly, forgetting that they have been generated through Łukasiewicz implication, and where the levels would only be regarded as ordered.

\subsection{Inference principle}

Assume we have three vectors $\left(a_{1}, \ldots, a_{n}, a_{n+1}\right)$, $\left(b_{1}, \ldots, b_{n}, b_{n+1}\right),\left(c_{1}, \ldots, c_{n}, c_{n+1}\right)$ providing complete descriptions of three situations according to $n+1$ variables, which are all valued in $[0,1]$ (or in a discrete subset of it including the bottom and top 


\begin{tabular}{c|cccc} 
& $a_{i}$ & $b_{i}$ & $c_{i}$ & $d_{i}$ \\
\hline 1 & 1 & 1 & 1 & 1 \\
2 & $\frac{1}{2}$ & $\frac{1}{2}$ & $\frac{1}{2}$ & $\frac{1}{2}$ \\
3 & 0 & 0 & 0 & 0 \\
4 & 1 & 1 & 0 & 0 \\
5 & $\frac{1}{2}$ & $\frac{1}{2}$ & 0 & 0 \\
6 & 0 & 0 & $\frac{1}{2}$ & $\frac{1}{2}$ \\
7 & $\frac{1}{2}$ & $\frac{1}{2}$ & 1 & 1 \\
8 & 1 & 1 & $\frac{1}{2}$ & $\frac{1}{2}$ \\
9 & 0 & 0 & 1 & 1 \\
10 & 1 & 0 & 1 & 0 \\
11 & 1 & $\frac{1}{2}$ & 1 & $\frac{1}{2}$ \\
12 & $\frac{1}{2}$ & 1 & $\frac{1}{2}$ & 1 \\
13 & 0 & $\frac{1}{2}$ & 0 & $\frac{1}{2}$ \\
14 & $\frac{1}{2}$ & 0 & $\frac{1}{2}$ & 0 \\
15 & $\frac{1}{2}$ & 1 & 0 & $\frac{1}{2}$ \\
16 & 0 & $\frac{1}{2}$ & $\frac{1}{2}$ & 1 \\
17 & 1 & $\frac{1}{2}$ & $\frac{1}{2}$ & 0 \\
18 & $\frac{1}{2}$ & 0 & 1 & $\frac{1}{2}$ \\
19 & 0 & 1 & 0 & 1
\end{tabular}

Table 2: The 19 cases where an analogical proportion perfectly holds in the tri-valued case

elements 0 and 1). Now consider that we have a fourth piece of data $d=\left(d_{1}, \ldots, d_{n}\right)$, which is incomplete in the sense that $d_{n+1}$ is unknown. In case this new piece of data forms an analogical proportion in a component-wise manner for each of the first $n$ variables, one may consider to apply an inductive step that amounts to assume that an analogical proportion still holds for the $(n+1)$ th variable. This corresponds to adopt the following general pattern

$$
\frac{\forall i \in[1, n], \quad a_{i}: b_{i}:: c_{i}: d_{i} \text { holds }}{a_{n+1}: b_{n+1}:: c_{n+1}: d_{n+1} \text { holds }}
$$

It simply means that if the known part of $d$ is in formal analogical proportion with the corresponding known parts of $a, b$ and $c$ then it should also be true for the unknown part of $d$. This is obviously a form of reasoning that is not sound, but which may be useful for trying to guess unknown values.

In order to do that, we should also be able to compute $d_{n+1}$ from the fact that $a_{n+1}: b_{n+1}:: c_{n+1}: d_{n+1}$ holds. In the Boolean case, the solution $x$ of an equation of the form $a: b:: c: x$ may not exist. Indeed, the patterns $1: 0:: 0: x$ and $0: 1:: 1: x$ have no solution. When the solution exists, it is unique and is given by $x=c \equiv(a \equiv b)$ as first suggested in [17] (see $[9])$. In the multiple-valued case, it can be checked that there exists an $x$ such that $a: b:: c: x$ holds at degree 1 if and only if $x=c+b-a \in[0,1]$ [13]. When it exists, the solution is unique. In this paper, we also use equations of the form $a: x:: x: b$ whose unique solution is given by $x=\frac{a+b}{2}$.

Let us consider an example (slightly modified from [9]) in order to illustrate the inference pro- cess. Assume a base of cases describing houses to let. In the example, we consider three features: nature (villa (1) or apartment (0)), air conditioning (equipped (1) or not (0)), and price (cheap (1) or expensive (0)). Assume we know the three cases:

$$
\begin{aligned}
& a=\text { (villa, equip., expen.) }=(1,1,0) \\
& b=\text { (villa, not-eq., cheap) }=(1,0,1) \\
& c=\text { (apart., equip., expen.) }=(0,1,0)
\end{aligned}
$$

Assume now a fourth house described by $d=$ (apart., not-eq., $x)=(0,0, x)$ for which one has to guess its price category. After checking that for the first two variables we have an analogical proportion between $a, b, c$, and $d$ (indeed in terms of truth values we have $(1: 1:: 0: 0)$, and $(1: 0:: 1: 0)$ ), one may assume that it also holds for the other components and the unique solution of the equation $(0: 1$ :: $0: x)=1$ is $x=1$, which means "cheap" here.

A refined version of the example can be described in a graded manner as e.g., $a=(1,1,0)$, $b=\left(1,0, \frac{1}{2}\right), c=(0,1,0)$, where the degrees may stand for the extent to which the price is cheap. Using Lukasiewicz implication, one gets $d=\left(0,0, \frac{1}{2}\right)$, i.e. the price of $d$ should be between cheap and expensive.

Some comments are in order. First, this example illustrates the fact that the inference mechanism is more productive than the one underlying standard case-based reasoning [18], as pointed out in [19, 9], since $d$ is not similar to any of $a, b$, and $c$, strictly speaking. Rather, it exhibits an adaptation mechanism that exploits the differences between the cases put in parallel. Besides, if we see the $(n+1)$ th variable as referring to a class, and the $n$ other variables as attributes describing situations in terms of possibly relevant features, we are faced with a classification problem, i.e. a learning-like task where a new item has to be classified on the basis of available examples. This idea first discussed in [10], has given encouraging results on preliminary examples [20]. The rule completion problem that we consider in this paper, and that we handle with this approach in the next section, is slightly different in the sense that we may assume that all the variables are relevant for predicting the conclusion value (since they appear in the rules available in the base). This is not the case in classification problems where some components of the description vectors may be irrelevant, and thus need not be involved in analogical proportions. Moreover, in the classification problem, analogical proportions that are approximately true (i.e., true to a degree less than 1) may be acceptable, while in the following we are going to require perfect analogical proportions.

\section{Extrapolating missing rules}

We are now in position to apply the analogical proportion based inference mechanism to the comple- 
tion of a set of rules. Let us first consider some examples, before laying bare the underlying principle.

\subsection{Examples}

Example 1. Assume we know the two rules

rule 1 "if $X_{1}$ is small and $X_{2}$ is small then $Y$ is large"

rule 2 "if $X_{1}$ is small and $X_{2}$ is large then $Y$ is small"

where the possible labels associated with variables $X_{1}, X_{2}$, and $Y$ are small, medium, or large. Then, if we wonder what may be a plausible conclusion for the rule

rule 3 "if $X_{1}$ is small and $X_{2}$ is medium then $Y$ is ..."

we may observe that a kind of analogical proportion of the form rule 1 : rule 3 :: rule 3 : rule 2 holds. Indeed, one may consider that we have for variable $X_{1}$ : small : small :: small : small, which certainly holds on the basis of pure identity, and for variable $\mathrm{X}_{2}$ we get small : medium :: medium : large, which holds as much as the increase from "small" to "medium" is the same as the increase from "medium" to "large". In fact, this sounds just like the analogical proportions $0: 0:: 0: 0$ (for variable $X_{1}$ ) and $0: \frac{1}{2}:: \frac{1}{2}: 1$ (for variable $X_{2}$ ) encountered in the previous section. On this basis, one may conclude for rule 3 that " $Y$ is medium" since large : medium :: medium : small should hold as well. Indeed, it can be checked that the unique solution of $1: x:: x: 0$ is $x=\frac{1}{2}$; see e.g. Table 2 .

Let us now consider a modified version of the previous example.

Example 2. Assume the two rules are now:

rule 1 "if $X_{1}$ is large and $X_{2}$ is small then $Y$ is large"

rule 2 "if $X_{1}$ is small and $X_{2}$ is large then $Y$ is small"

Looking for a plausible conclusion for the rule

rule 3 "if $X_{1}$ is medium and $X_{2}$ is medium then $Y$ is ..."

on the basis of the analogical proportion rule 1 : rule 3 :: rule 3 : rule 2 , which corresponds for variable $X_{1}$ to $0: \frac{1}{2}:: \frac{1}{2}: 1$, and for variable $X_{2}$ to $1: \frac{1}{2}:: \frac{1}{2}: 0$, leads again to the conclusion " $Y$ is medium" (by solving an equation of the form $1: x:: x: 0)$.

Clearly, more than 3 rules may be involved as in the following example:
Example 3 Assume we have the following rules in the base:

rule 1 "if $X_{1}$ is small and $X_{2}$ is large then $Y$ is large"

rule 2 "if $X_{1}$ is small and $X_{2}$ is small then $Y$ is small"

rule 3 "if $X_{1}$ is large and $X_{2}$ is large then $Y$ is large"

and we look for a plausible conclusion for the rule

rule 4 "if $X_{1}$ is large and $X_{2}$ is small then $Y$ is ..."

Then, the analogical inference leads to $Y$ is small", on the basis of the patterns $0: 0:: 1: 1$ and $1: 0:: 1: 0$.

\subsection{Principle and assumptions}

In the above examples, several assumptions are implicitly made. First, the domain of the variables is a discrete, totally ordered set of labels. In the examples, small is indeed smaller than medium, itself smaller than large. Moreover, it is also tacitly assumed that the distance between two successive labels is always the same, in order to make the the domain of the variables isomorphic to scales such as $\{0,1\},\left\{0, \frac{1}{2}, 1\right\},\left\{0, \frac{1}{3}, \frac{2}{3}, 1\right\},\left\{0, \frac{1}{4}, \frac{1}{2}, \frac{3}{4}, 1\right\}$, $\left\{0, \frac{1}{5}, \frac{2}{5}, \frac{3}{5}, \frac{4}{5}, 1\right\}$ and so on. Then, it becomes possible to compare differences between two labels. Note also that it is not required to use the same scale for each variable. Besides, one may insert abstract labels in the scale, if one wants to express that some labels are not at equal distance from their predecessor and their successor.

Second, it is also assumed that the mapping that relates the variables $X_{1}, \ldots, X_{n}$ to $Y$ is monotonically increasing (or decreasing) w.r.t. each variable. While this puts a theoretical restriction on the kind of rule bases that may be considered, the assumption seems to be satisfied when the rules model generic knowledge (rather than factual knowledge such as observations or specifications), and the conditions that appear are sufficiently atomic. Moreover, if the mapping is not monotonic, it can usually be made monotonic, e.g. by refining the conditions or by adding appropriate context information. It can be shown that the analogical inference mechanism preserves monotonicity. In particular, if the labels in the condition part of the incomplete rule are between the labels of two other rules for each variable (with respect to the ordering associated with its domain), then this also holds for the labels in the conclusions.

The analogical inference mechanism enforces that the same amount(s) of increase (or decrease) on the 
$X_{i}$ 's w.r.t. any value always has the same amount of effect on $Y$. For instance, in Example 1, the amount of increase from small to medium being the same from medium to large for $X_{2}$, it should lead to similar decreases for $Y$ (from large to medium, and from medium to small). Still, the inference in Example 2, may be found more adventurous than in Example 1, since in this latter example, the variation of only one variable causes the variation of $Y$, the other variable remaining constant, while in Example 2, there are simultaneous variations of $X_{1}$ and $X_{2}$.

Note that this assumption of linearity essentially means that, in absence of further information, we complete the knowledge base in a way which is as simple as possible, and therefore as natural as possible. Also note that while the mapping underlying the rule base is assumed to be linear w.r.t. the numerical representation of the labels, it is not required that the mapping is linear w.r.t. any underlying quantitative domains. For example, matching "cheap", "moderate" and "expensive" with a 3-level scale in an analogical proportion pattern does not necessarily mean that we consider the actual price difference between cheap and moderate prices to be equal to the actual price difference between moderate and expensive prices (as already suggested at the end of section 2.2).

It should also be noticed that the analogical inference cannot always produce a result. For instance, in Example 2, from

rule 1 "if $X_{1}$ is large and $X_{2}$ is small then $Y$ is large"

rule 2 "if $X_{1}$ is small and $X_{2}$ is large then $Y$ is small"

nothing can be concluded for a rule of the form

rule 3 "if $X_{1}$ is large and $X_{2}$ is medium then $Y$ is ..."

which would rather require to know a rule with conditions

"if $X_{1}$ is large and $X_{2}$ is large".

Still, it is possible to get different conclusions when the number of rules is large enough, as shown on the following abstract example.

Example 4 Assume we have 5 rules relating $X_{1}, X_{2}, X_{3}$ to $Y$, corresponding to the following vectors $(1,1,1,1),\left(\frac{1}{2}, 1,1,1\right),(1,0,1,1),\left(\frac{1}{2}, 0,1, \frac{1}{2}\right)$, and $(0,1,1,1)$. Note that the 5 rules do not violate the monotonicity condition. Then from $(1,1,1,1),(1,0,1,1)(0,1,1,1)$, we conclude $x=1$ for the incomplete rule $(0,0,1, x)$, while from $(1,1,1,1),\left(\frac{1}{2}, 0,1, \frac{1}{2}\right),\left(\frac{1}{2}, 1,1,1\right)$, we get $x=\frac{1}{2}$ for the incomplete rule $(0,0,1, x)$. In such a case, there is no apparent reason to prefer a conclusion to the other, and it is advisable to conclude that $\left(x=\frac{1}{2}\right) \vee(x=1)$, i.e., for instance, $Y$ is medium or large.

The fact that several distinct conclusions may be obtained should not be seen as a crippling defect. As said in the previous section, the analogical proportion-based inference mechanism cannot in general guarantee sound conclusions. This is already true for case-based reasoning. So we may have two kinds of attitude:

- either we severely restrict the application of the inference mechanism to particular patterns for which the inference may become sound under strong hypotheses like the linearity of the underlying mapping. In such a case, cautious results are obtained, but which are guaranteed to be sound with respect to some natural semantics.

- or we allow for a more extensive use of the inference mechanism, and one may only obtain defeasible, and sometimes multiple, conclusions. In case of multiple conclusions, combining them disjunctively is natural inasmuch as they are all possible. Note that such an optimistic strategy is common in approaches to non-monotonic reasoning as well as information fusion.

\section{Further remarks and extensions}

In the previous section, we have outlined a method for completing a rule base by enforcing analogical proportions. Some further remarks are worth mentioning regarding some specific aspects of the approach.

\subsection{Iteration of the completion process}

First, the inference process may have to be iterated for getting an answer to some completion query. This is the case in the following example, where the rule base includes the three rules:

- "If $X_{1}$ is small and $X_{2}$ is medium then $Y$ is medium" (1)

- "If $X_{1}$ is large and $X_{2}$ is medium then $Y$ is medium" (2)

- "If $X_{1}$ is medium and $X_{2}$ is small then $Y$ is large" (3)

Suppose we want to complete the rule

- "If $X_{1}$ is medium and $X_{2}$ is large then $Y$ is ???"

The conclusion cannot be obtained directly. To get it, however, we might first derive the following rule

- "If $X_{1}$ is medium and $X_{2}$ is medium then $Y$ is medium" (4)

since (1):(4)::(4):(2) holds, and then we get the solution

- "If $X_{1}$ is medium and $X_{2}$ is large then $Y$ is small" (5)

since (3):(4)::(4):(5) holds also. 


\subsection{Making use of explicit monotonicity information}

We may also think of supplementing the analogical inference by exploiting the information on the monotonicity of the underlying mapping. For instance, if we only have the two rules "If $X$ is very small then $Y$ is very small" and "If $X$ is small then $Y$ is small", one may conclude in case where " $X$ is large" at least that " $Y$ is small $\vee$ medium $\vee$ large $\vee$ very large", if the mapping is increasing and if we work with five labels ("very small", "small", ..., "very large"). Note that here the use of disjunction acknowledges the imprecision of the result rather than referring to conflicting conclusions as above in the previous section.

\subsection{Finite sets of labels on continuous domains}

The proposed approach supposes that we are working with discrete domains, i.e. the labels are viewed as distinct granules. In case of continuous domains, one may need to view the labels as intervals rather than as discrete points. For instance, consider the variable "price" with $[0,1]$ as normalized domain, and the four labels "cheap", "moderate", "expensive", "exorbitant". These four labels may be associated with sub-intervals such as $[0, \alpha]$, $[\alpha, \beta],[\beta, \gamma],[\gamma, 1]$. Suppose we have the equation cheap : moderate :: moderate : $x$. Applying the equation $x=c+b-a$ to the intervals yields $x=2 \times[\alpha, \beta]-[0, \alpha]=[\alpha, 2 \beta]$. If $\beta$ is taken to be equal to $\frac{1}{2}$, we get the conclusion $x=[\alpha, 1]$, i.e. $x=$ moderate $\vee$ expensive $\vee$ exorbitant, which is a more cautious conclusion than the one obtained by solving the equation

$$
\text { cheap : moderate :: moderate : } x
$$

at the label level, which gives $x=$ expensive. Thus, the compatibility with continuous domains may lead to prefer more imprecise conclusions.

One might even go one step further by considering fuzzy set-valued analogical proportions, rather than interval-valued analogical proportions as above. Then, we would obtain a fuzzy set representation for $x$ (using the extension principle), which would have to be linguistically approximated in terms of the label vocabulary. However, this seems too sophisticated to be useful in practice.

\subsection{Softening the induction step}

The fact that analogical proportion equations yield precise solutions (at least for discrete universes) may sometimes look too adventurous in our rule completion problem. Indeed, suppose we use the labels $\{$ very-small, small, medium, large, very-large $\}$, then completing the two rules "If $X$ is very-small then $Y$ is very-small" and "If $X$ is very-large then $Y$ is very-large" by "If $X$ is medium then $Y$ is medium" may seem quite precise: one might consider that " $Y$ is small $\vee$ medium $\vee$ large" would be more realistic. One way of implementing such a strategy would be to replace the precise solution of the analogical proportion equation by a possibility distribution, whose core contains the conclusions that can be obtained from perfect solutions to analogical proportions. The support of the possibility distribution would then also contain those labels that can be obtained as approximate solutions of the equation, i.e. those solutions which give a truth-value less than 1 (but strictly greater than some threshold) to the analogical proportion. In this way, we implicitly take into account the fact that e.g. the change from very-small to small may not be exactly the same as the change from small to medium, as well as the fact that some small irregularities are somewhat plausible to occur.

\section{Concluding remarks}

Fuzzy set theory offers a quantitative framework for approximate reasoning [21], interpolative reasoning $[22,23,4,3,5]$, and similarity-based reasoning. Thus, [24] adapts the solution of an already solved situation to a similar new situation according to the degree of resemblance between these two situations. These approaches take advantage of fuzzy set membership function based representations.

In this paper, since such representations are not always available (especially when we are not on unidimensional numerical universes), we have followed another road for interpolating or extrapolating new rules from a rule base. We have outlined a new approach where a new rule is inferred from two or three other rules under conditions expressed in terms of analogical proportions. This approach is qualitative, and may in some sense be viewed as an attempt at computing with words [6] since it can be processed at a purely symbolic level.

Another qualitative approach to the same problem has recently been proposed by the authors [25], using the notion of betweenness as primitive in the setting of Gärdenfors conceptual spaces. In this latter approach, the basic inference pattern involves two rules, while in the approach presented here, it relies on three rules (which is also to be compared with case-based reasoning where a current situation is matched with only one rule at a time).

Besides, the proposed approach completes the partial knowledge about a mapping on the basis of a generic principle of regularity which is enforced through the satisfaction of analogical proportions, just as a least commitment principle is used in [26] for completing preferences between multiple criteria evaluations while taking into account the monotonicity of the underlying evaluation process and the relative importance of criteria. 


\section{References}

[1] G. Brewka. Nonmonotonic Reasoning: Logical Foundations of Commonsense. Cambridge University Press, 1991.

[2] S. Benferhat, D. Dubois, and H. Prade. Nonmonotonic reasoning, conditional objects and possibility theory. Artificial Intelligence, 92(12):259-276, 1997.

[3] B. Bouchon-Meunier, F. Esteva, L. Godo, M. Rifqi, and S. Sandri. A principled approach to fuzzy rule base interpolation using similarity relations. In Proc. of the EUSFLAT-LFA Joint Conference, Barcelona, pages 757-763.

[4] L. Ughetto, D. Dubois, and H. Prade. Fuzzy interpolation by convex completion of sparse rule bases . In Proc. 9th IEEE Int.Conf. on Fuzzy Systems (FUZZ-IEEE'00), San Antonio (Texas), May, 7-10, pages 465-470, 2000.

[5] I. Perfilieva, D. Dubois, H. Prade, F. Esteva, L. Godo, and P. Hod'áková. Interpolation of fuzzy data: Analytical approach and overview. Fuzzy Sets and Systems, in press, 2011.

[6] L. A. Zadeh. Fuzzy logic = computing with words. IEEE Transactions on Fuzzy Systems, 4 (2):103-111, 1996.

[7] D. G. Schwartz. A system for reasoning with imprecise linguistic information. Int. J. $A p$ prox. Reasoning, 5:463-488, 1991.

[8] D. Dubois, H. Prade, L. Foulloy, and S. Galichet. Performing approximate reasoning withs words. In L. Zadeh and J. Kacprzyk, editors, Computing with Words in Information/Intelligent Systems 1, pages 2449. Springer-Verlag, 1999.

[9] L. Miclet and H. Prade. Handling analogical proportions in classical logic and fuzzy logics settings. In Proc. 10th ECSQARU, Verona, volume LNCS 5590, pages 638-650. Springer, 2009.

[10] H. Prade and G. Richard. Reasoning with logical proportions. In Proc. 12th Inter. Conf. on Principles of Knowledge Representation and Reasoning, KR 2010, Toronto, May 9-13, (F. Z. Lin, U. Sattler, M. Truszczynski, eds.), pages 545-555. AAAI Press, 2010.

[11] Y. Lepage. De l'analogie rendant compte de la commutation en linguistique. http://www. slt.atr.jp/ lepage/pdf/dhdryl.pdf, Grenoble, 2003. Habilitation à diriger des recherches.

[12] N. Stroppa and F. Yvon. Analogical learning and formal proportions: Definitions and methodological issues. Technical Report ENST-2005-D004, http://www.tsi.enst.fr/ publications/enst/techreport-2007-6830.pdf, June 2005.

[13] H. Prade and G. Richard. Multiple-valued logic interpretations of analogical, reverse analogical, and paralogical proportions. In Proc. 40th IEEE Inter. Symp. on Multiple-Valued Logic,
ISMVL 2010, Barcelona, Spain, May 26-28, pages 258-263, 2010.

[14] L. Godo and C. Sierra. A new approach to connective generation in the framework of expert systems using fuzzy logic. In Proc. 18th Inter. Symp. Multiple-Valued Logic, Palma, pages 157-162, 1988.

[15] M. Mas, G. Mayor, and J. Torrens. t-operators and uninorms on a finite totally ordered set. Int. J. Intelligent Systems, 14:909-922, 1999.

[16] J. Fodor. Smooth associative operations on finite ordinal scales. IEEE Trans. on Fuzzy Systems, 8:791-795, 2000.

[17] S. Klein. Culture, mysticism and social structure and the calculation of behavior. In European Conference on Artificial Intelligence (ECAI-82), Orsay, France, pages 141-146, 1982.

[18] A. Aamodt and E. Plaza. Case-based reasoning: Foundational issues, methodological variations, and system approaches. Artificial Intelligence Communications, 7(1):39-59, 1994.

[19] H. Prade. Proportion analogique et raisonnement à partir de cas. In Rencontres Francophones sur la Logique Floue et ses Applications, pages 296-303. Cépaduès, 2008.

[20] H. Prade, G. Richard, and B. Yao. Classification by means of fuzzy analogy-related proportions - A preliminary report. In Proc. 2nd IEEE International Conference of Soft Computing and Pattern Recognition (SoCPaR 2010), Cergy-Pontoise, Dec. 7-10, pages 297$302,2010$.

[21] L. A. Zadeh. A theory of approximate reasoning. In J. E. Hayes, D. Mitchie, and L. L. Mikulich, editors, Machine intelligence, Vol. 9, pages 149-194. Wiley, 1994.

[22] H. Prade. Some issues in approximate and plausible reasoning in the framework of a possibility theory-based approach. In L. M. Vaina, editor, Matters of Intelligence, pages 263-287. D. Reidel, 1987.

[23] D. Dubois, F. Esteva, P. Garcia, L. Godo, and H. Prade. A logical approach to interpolation based on similarity relations. Int. J. of Approximate Reasoning, 17:1-36, 1997.

[24] B. Bouchon-Meunier and L. Valverde. A fuzzy approach to analogical reasoning. Soft Computing, 3:141-147, 1999.

[25] S. Schockaert and H. Prade. Qualitative reasoning about incomplete categorization rules based on interpolation and extrapolation in conceptual spaces. Submitted.

[26] R. Gérard, S. Kaci, and H. Prade. Ranking alternatives on the basis of generic constraints and examples - a possibilistic approach. In M. M. Veloso, editor, Proc. 20th Inter. Joint Conf. on Artificial Intelligence (IJCAI 2007), Hyderabad, India, Jan. 6-12, pages 393-398, 2007. 